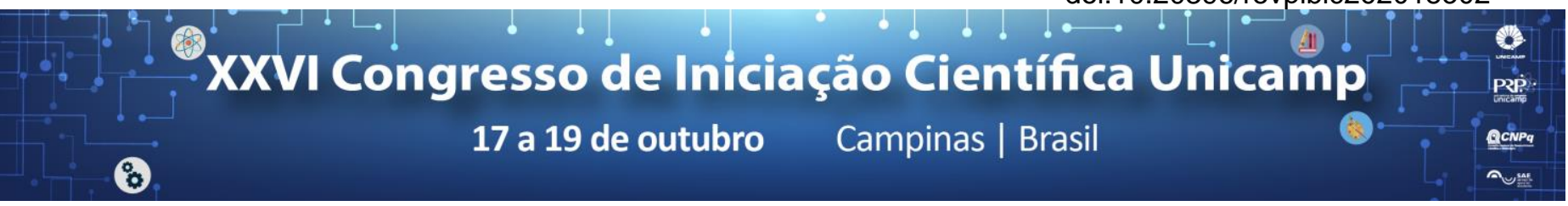

\title{
A WSN prototype for Energy Consumption Monitoring
}

\author{
Cássio G. Dezotti, Fernanda A. Machado, Regina C. Ruschel.
}

\begin{abstract}
This paper presents a wireless sensor network prototype developed for the Internet of Things (IOT) paradigm to monitor and characterize energy consumption issues in an institutional building. The prototype comprises sensing, computing and communicating elements to enhance facility managers' problem-solving over energy management.
\end{abstract}

\section{Key words: \\ Energy Management, Wireless Sensor Network, Operation and Maintenance.}

\section{Introduction}

Energy consumption in Brazil is expected to increase mainly in institutional and commercial buildings [1]. Such a bias is part of a global issue and demand from building owners the adoption of measures concerning Energy Management and its related long-term costs. Regarding that, the University of Campinas is concerned on spending around 25 million reais per year with electricity [2], which points out the need of Energy Management Systems (EMS) in its facilities. Considering such need in University' buildings, the primary aim of this research was to develop a WSN prototype to monitor and characterize energy consumption issues in an institutional building. As a proof of concept, the prototype was designed to monitor the lighting system of a research laboratory.

\section{Development and Discussion}

The prototype consisted of a Wireless Sensor Network (WSN), an infrastructure that comprises sensing, computing and communication elements to provide a user the ability to manage and react to events in a smart environment [3]. Through the adoption of Design Science Research [4], the stages of design, development, and evaluation considered OpenEnergyMonitor guidelines. In the design phase, we assessed the performance of a range of electronic components before building the actual artifact. As a result, we chose a NodeMCU microcontroller for prototyping, since it already had an ESP8266 Wi-Fi chip embedded. For achieving an artifact that covered the entire room's lighting system, we developed and tested two modules for the NodeMCU. The first one consisted of pull-up and pull-down resistors, a capacitor, a TC4051 Cl and six SCT-013-020 AC sensors. The TC4051 $\mathrm{Cl}$ amplified the analog entries capabilities of the NodeMCU to embrace the AC sensors - one for each existing lighting fixture. The SCT-013 family of sensors were non-invasive and allowed prototype's implementation without any intrusion in the monitored lighting system. Those sensors went through calibration procedures. The second module used a $9 \mathrm{~V}$ AC-AC adapter, a capacitor, as well as pull-up, pull-down and burden resistors to measure the phase voltage of the room. We developed a schematic that linked both modules to a single NodeMCU microcontroller, a low-cost and effective solution (Image 1 - left). Then, we established a connection via Wi-Fi protocol between NodeMCU and the room' routing to enable the transmission of the collected data to ThingSpeak. ThingSpeak is an open-source loT platform that hosts a cloud database, which allowed the aggregation, view, and analysis of real-time collected data.
With a script and native field parameters, the current and voltage data was transformed into power consumption information. The information embraced the room's lighting system status (on/off), power consumption in real-time and its averages through periods of time (current, hourly, daily...). Those outputs allowed the lighting system' performance and associated costs analysis, in a disaggregated strategy (per room, circuit and lighting fixture). Besides, they addressed a demand for condition monitoring, aiming at predictive maintenance and consumption decrease. Such information in JSON format integrated an interactive web interface for improved feedback. After a pilot implementation, the prototype was evaluated by controlled experiments. This procedure identified real failures in the lighting system. Other electrical equipment when turned on influenced its performance. We corrected condition-monitoring ranges after that and built the final artifact on a pre-drilled circuit board for permanent installation (Image 1 - right).
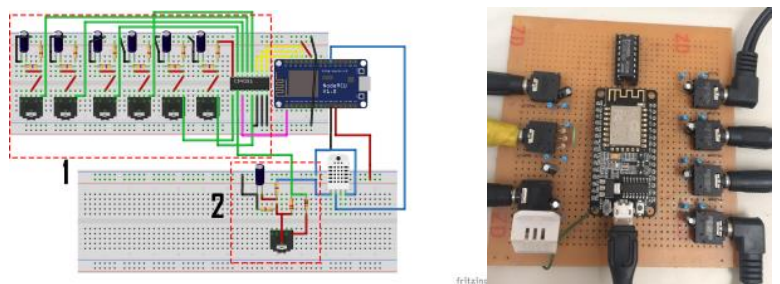

Image 1. Schematic (left), the final artifact (right).

\section{Conclusions}

The prototype promoted the monitoring and enabled the analysis of the lighting system's energy use in real-time. It combined concepts of WSN, IoT, and computational programming in a low-cost and scalable solution. Such a solution may cover the whole building in attendance to energy management and facility managers' needs.

\section{Acknowledgement}

This research was supported by PIBIC and CAPES Scholarships in partnership with LAMPA (Laboratory of Architecture, Design Methodology and Automation) at FEC-UNICAMP.

${ }^{1}$ Ministério de Minas e Energia - Empresa de Pesquisa Energética. Demanda de Energia 2050: Nota Técnica DEA 13/15. Brasília:2016.

${ }^{2}$ I. Gardenal. Unicamp e CPFL celebram parceria em eficiência energética e redução de consumo de energia. Disponível em:

$<h$ ttps://www.unicamp.br/unicamp/noticias/2018/01/31/unicamp-e-cpflcelebram-parceria-em-eficiencia-energetica-e-reducao-de-consumo $>$. Acesso em: 01 mar. 2018

${ }^{3}$ Sohraby, K.; Minoli, D.; Znati, T. Wireless Senor Network Technology, Protocols and Applications. 2007.

${ }^{4}$ Dresch, A. et al. Design Science Research: método de pesquisa para avanço da ciência e tecnologia. ed: Bookman, Porto Alegre, 2015. 